\title{
OPEN Strain specific motility patterns and surface adhesion of virulent and probiotic Escherichia coli
}

\author{
M. M. Abdulkadieva ${ }^{1,2}$, E. V. Sysolyatina ${ }^{3 凶}$, E. V. Vasilieva ${ }^{1,3}$, A. I. Gusarov ${ }^{4}$, P. A. Domnin ${ }^{3}$, \\ D. A. Slonova ${ }^{5,6}$, Y. M. Stanishevskiy ${ }^{2}$, M. M. Vasiliev $^{1}$, O. F. Petrov ${ }^{1}$ \& S. A. Ermolaeva ${ }^{3}$
}

Bacterial motility provides the ability for bacterial dissemination and surface exploration, apart from a choice between surface colonisation and further motion. In this study, we characterised the movement trajectories of pathogenic and probiotic Escherichia coli strains (ATCC43890 and M17, respectively) at the landing stage (i.e., leaving the bulk and approaching the surface) and its correlation with adhesion patterns and efficiency. A poorly motile strain JM109 was used as a control. Using specially designed and manufactured microfluidic chambers, we found that the motion behaviour near surfaces drastically varied between the strains, correlating with adhesion patterns. We consider two bacterial strategies for effective surface colonisation: horizontal and vertical, based on the obtained results. The horizontal strategy demonstrated by the M17 strain is characterised by collective directed movements within the horizontal layer during a relatively long period and nonuniform adhesion patterns, suggesting co-dependence of bacteria in the course of adhesion. The vertical strategy demonstrated by the pathogenic ATCC43890 strain implies the individual movement of bacteria mainly in the vertical direction, a faster transition from bulk to near-surface swimming, and independent bacterial behaviour during adhesion, providing a uniform distribution over the surface.

Flagella-dependent motility characteristics of many bacterial species are important features providing effective dissemination and occupation of novel environments. Flagella themselves provide motility and are also involved in interactions with surfaces, performing the initial steps in surface adherence and biofilm formation. Some pathogenic species use flagella for host tissue colonization ${ }^{3-6}$.

According to the model proposed by Misselwitz et al., interactions of motile bacteria with the cell surface are carried out in four stages: (1) landing (exit of the bacterium from the bulk), (2) near-surface swimming (NSS), (3) stopping (interactions between bacterial receptors and the surface), and (4) taking off (if the interactions are weak) or adhesion, which happens rarely ${ }^{7}$. Experimental observations on widely used model microorganisms, such as the Gram-negative bacterium Escherichia coli, established that trajectories of bacterial movement also depend on the distance from the solid surface. At a distance of 20-120 $\mu \mathrm{m}$ ('bulk'), about $70 \%$ bacteria show 'run and tumble' trajectories that are a number of broken lines, and movement of the rest $30 \%$ is characterized by smoother trajectories with smaller angles of rotation, named 'slow random walk's. Bacteria that locate closer to the glass at a distance of equal or less than $20 \mu \mathrm{m}$, i.e., at the «landing» stage, rotate ('tumble') less often, and their reorientation angles are about $34 \%$ smaller than the bacteria located in the bulk of the suspension. The direction of bacterial movement at a distance of $10-20 \mu \mathrm{m}$ is often parallel to the surface ${ }^{8}$. Motile species at close vicinity to the surface (a distance of less than $5 \mu \mathrm{m}$ ) demonstrate near-surface swimming that includes such a typical trajectory as long circular movement.

Many authors have suggested theoretical models of the described trajectories to provide arguments for different movement types ${ }^{9-11}$. To explain the biological sense of the 'unproductive' NSS circular motion demonstrated by the enterohemorrhagic E. coli O157:H7 strain, Ipina et al. proposed a mathematical model based on short-term stops and attachment of bacteria at various points of the trajectory, important for effective surface colonisation

${ }^{1}$ Department of Dusty Plasma, Joint Institute for High Temperatures, Russian Academy of Sciences, Moscow, Russia. 'Peoples' Friendship, University of Russia (RUDN University), Moscow, Russia. ${ }^{3}$ Laboratory of Ecology of Pathogenic Bacteria, N. F. Gamaleya National Research Centre of Epidemiology and Microbiology, Ministry of Health of the Russian Federation, Moscow, Russia. ${ }^{4}$ National Research University "Moscow Power Engineering Institute", Moscow, Russia. "Laboratory of Molecular Microbiology, Center of Life Sciences, Skolkovo Institute of Science and Technology, Moscow, Russia. ' Laboratory of Molecular Immunology, Dmitry Rogachev National Medical Research Center of Pediatric Hematology, Oncology and Immunology, Russian Ministry of Health, Moscow, Russia. ${ }^{\square}$ email: elena.sysolyatina84@gmail.com 


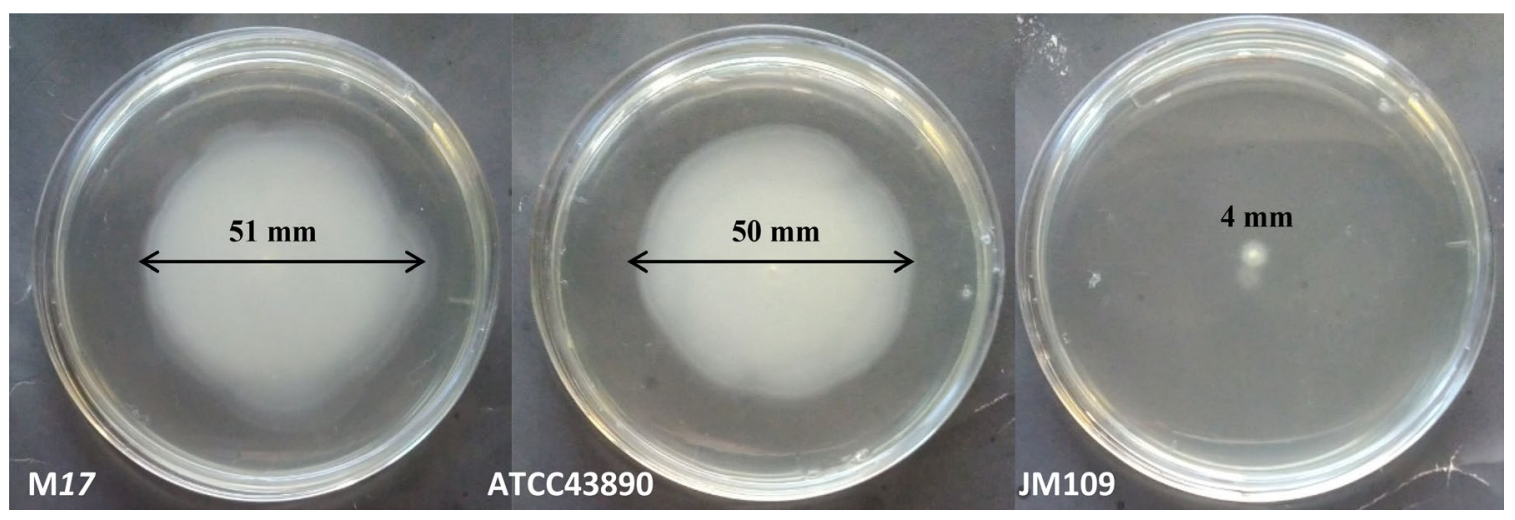

Figure 1. Motility of E. coli strains in a semi-solid agar.

and, consequently, survival in the environment and inside the host ${ }^{12}$. Other researchers have demonstrated that bacteria can interact with surfaces through 'head' or 'tail' collisions, the latter being more effective for adhesion ${ }^{13}$.

Bacterial movement has been studied in different bacterial strains and species, and although general movement patterns describe a majority of observations, a particular movement style may prevail in one bacterial species but can be rare in another. Meanwhile, bacteria are highly diverse, and the differences between strains within a single species might substantially affect motility. This interspecies difference is important for understanding the mechanisms underlying the strain-specific potential to survive within specific ecological niches. In particular, strains of well-known species such as E. coli are morphologically and physiologically very different. For example, the laboratory strain of E. coli JM109 is flagellated and forms biofilms well, but it is poorly motile due to a deletion of the recA gene, necessary for standard flagellar rotation switching ${ }^{14-17}$. Virulent toxin-producing E. coli strains belonging to the serotype O157:H7 are flagellated, motile, and successfully colonise the host intestine but are poor biofilm producers under experimental conditions ${ }^{18-21}$ Comparison of different strains under the same conditions is required to understand how motility patterns affect bacterial strain-specific potential to colonise different surfaces. In this study, we compared movement trajectories of pathogenic and probiotic E. coli strains (ATCC43890 and M17, respectively) at the landing stage (i.e., leaving the bulk and approaching the surface) and its correlation with adhesion patterns and efficiency.

\section{Experimental results}

Probiotic and virulent E. coli strains demonstrated similar macroscopic motility. Three E. coli strains were used, including the motile commensal probiotic strain M17, the motile pathogenic O157:H7 strain ATCC43890, and almost non-motile, but flagellated laboratory strain JM109 as a negative control $^{22}$.

The bacteriological motility test in semi-liquid agar did not reveal differences between the motile strains M17 and ATCC43890 (Fig. 1). Both strains spread in semi-liquid agar with similar effectiveness, which was noticeably higher than that of the control non-motile strain JM109. The opacity areas showing bacterial spreading in the semi-liquid agar after $24 \mathrm{~h}$ of growth were $4 \pm 0.5 \mathrm{~mm}$ for JM109, which could be regarded as a result of cell division (Fig. 1). The difference in opacity areas between M17 and ATCC43890 of $51 \pm 4 \mathrm{~mm}$ and $50 \pm 2 \mathrm{~mm}$, respectively, was insignificant $(p>0.05)$. Thus, the obtained results showed that the probiotic and virulent strains had the same macroscopic motility.

E. coli strains had different motility patterns. A microfluidic chamber was designed to study bacterial movement. The chamber had an area of $8 \times 10 \mathrm{~mm}$ and a height of $30 \mu \mathrm{m}$, covered with a glass slide (Fig. 2). The centre of the chamber was filled with a bacterial suspension with an $\mathrm{OD}_{600}$ of approximately 2.0.

We observed bacterial movement in the middle layer of the bacterial suspension, that is, at a distance of 10-15 $\mu \mathrm{m}$ from both the chamber bottom and the glass (Fig. 2a). This distance was used to ensure that the observed motion characterised the landing stage and was not due to bacterial interactions with the surface. To capture movement patterns, we recorded a set of short videos that were processed to obtain the main characteristics of movement (Fig. 3) and reconstruct trajectories within the layer of observation (Fig. 4). Based on the obtained data, we revealed differences in the movement patterns between strains.

Average values of velocity, distance and residence time (i.e., the time spent by an individual bacterium within the observed liquid layer) were calculated based on the results of $\sim 200$ reconstructed trajectories in $10 \mathrm{~s}$ video, taken for each strain (see Materials and Methods). Based on macroscopic observations, we suggested that the non-motile JM109 would demonstrate Brownian movement, while the movement of the two other strains would characterise the motion of motile bacteria showing parameters different from the non-motile strain.

Boxplot of typical bacterium velocity (Fig. 3 left) showed only a little difference between strains with mean velocity values of $5.93 \mu \mathrm{m} / \mathrm{sec}$ (CI 95\% 5.7-6.16), $6.49 \mu \mathrm{m} / \mathrm{s}$ (CI 95\% 6.26-6.72), and $6.36 \mu \mathrm{m} / \mathrm{s}$ (CI 95\% 5.93-6.79) for JM109, M17, and ATCC43890, respectively (Anova, F=4.56, $p<0.05$ ). At this, difference of mean values was significant only between JM109 and M17 (Tukey, HSD $=0.58, p<0.05$ ). While the speed values were almost similar for all three strains, other characteristics were strikingly different (Fig. 3, middle, right). The average distances, which individual bacterium ran within horizontal layer before leaving, were $3.17 \mu \mathrm{m}$ (CI 
a Microfluidic chamber

Top view

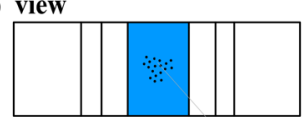

Side view Bacterial suspension

Cover glass

$30 \mu \mathrm{m}$

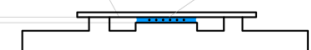

b
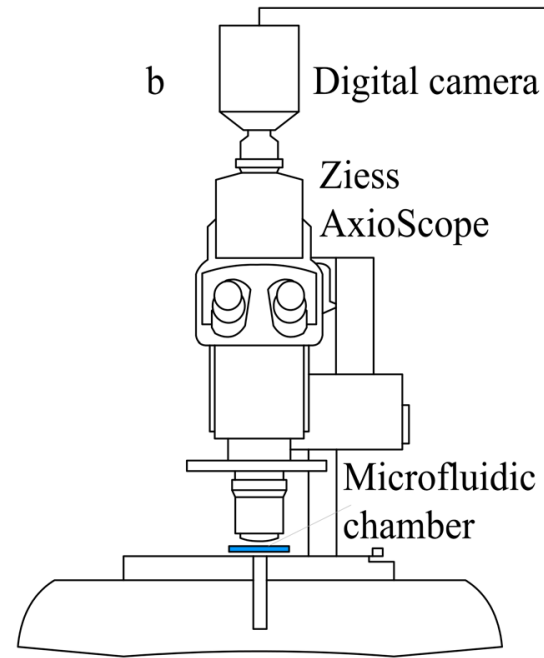

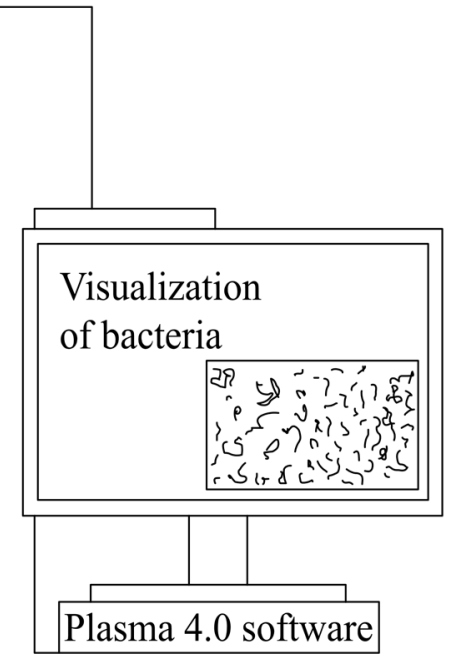

Figure 2. Scheme of (a) microfluidic chamber and (b) experimental setup.
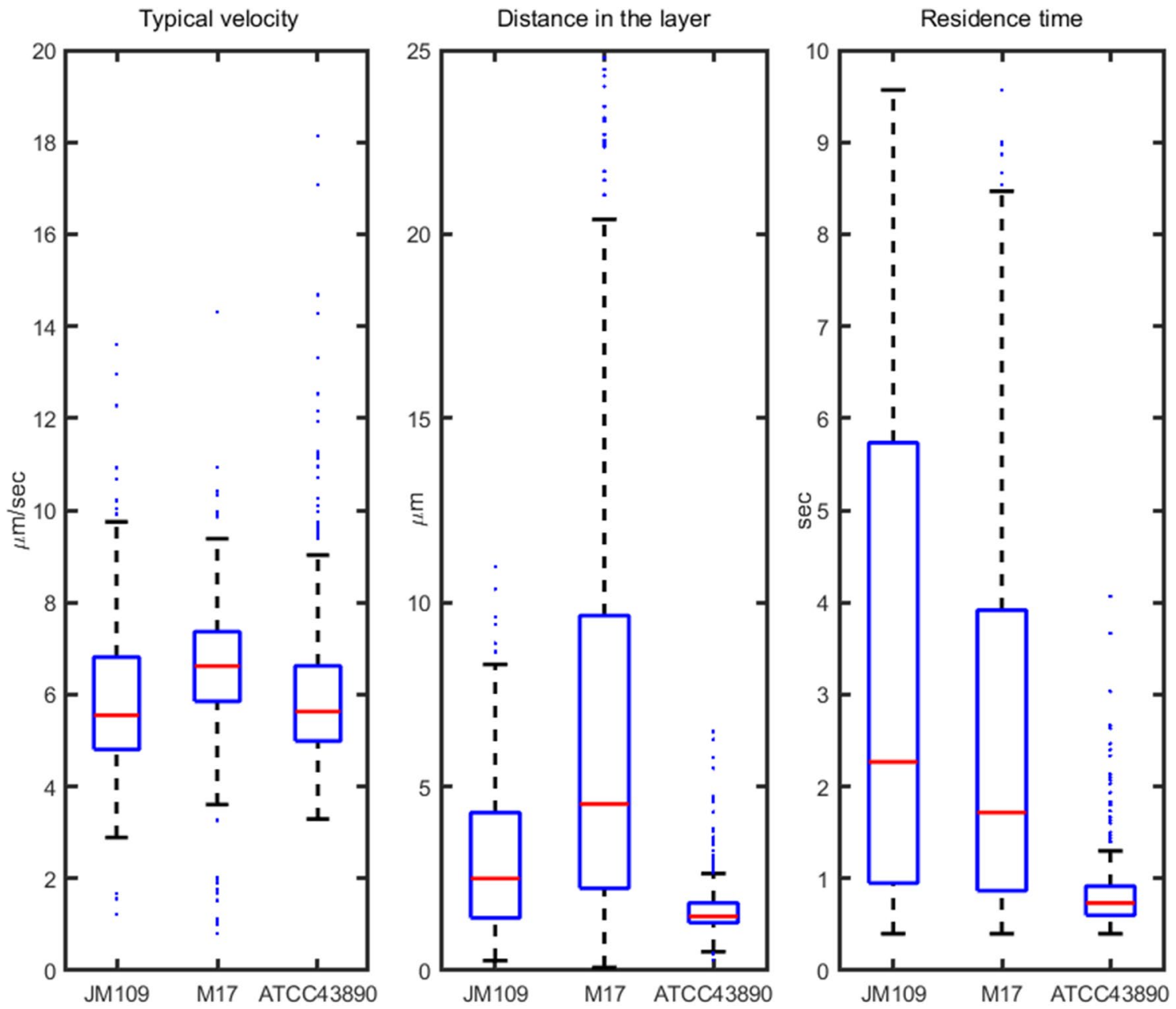

Figure 3. Boxplot with standard whiskers (1.5 IQR) of main characteristics of $E$. coli strains' movement: typical bacterium velocity (left), typical distance covered by bacteria within the layer (middle), typical residence time spent in the layer (right). Red lines-median values for characteristics. All distributions consist of $\sim 200$ trajectories. 

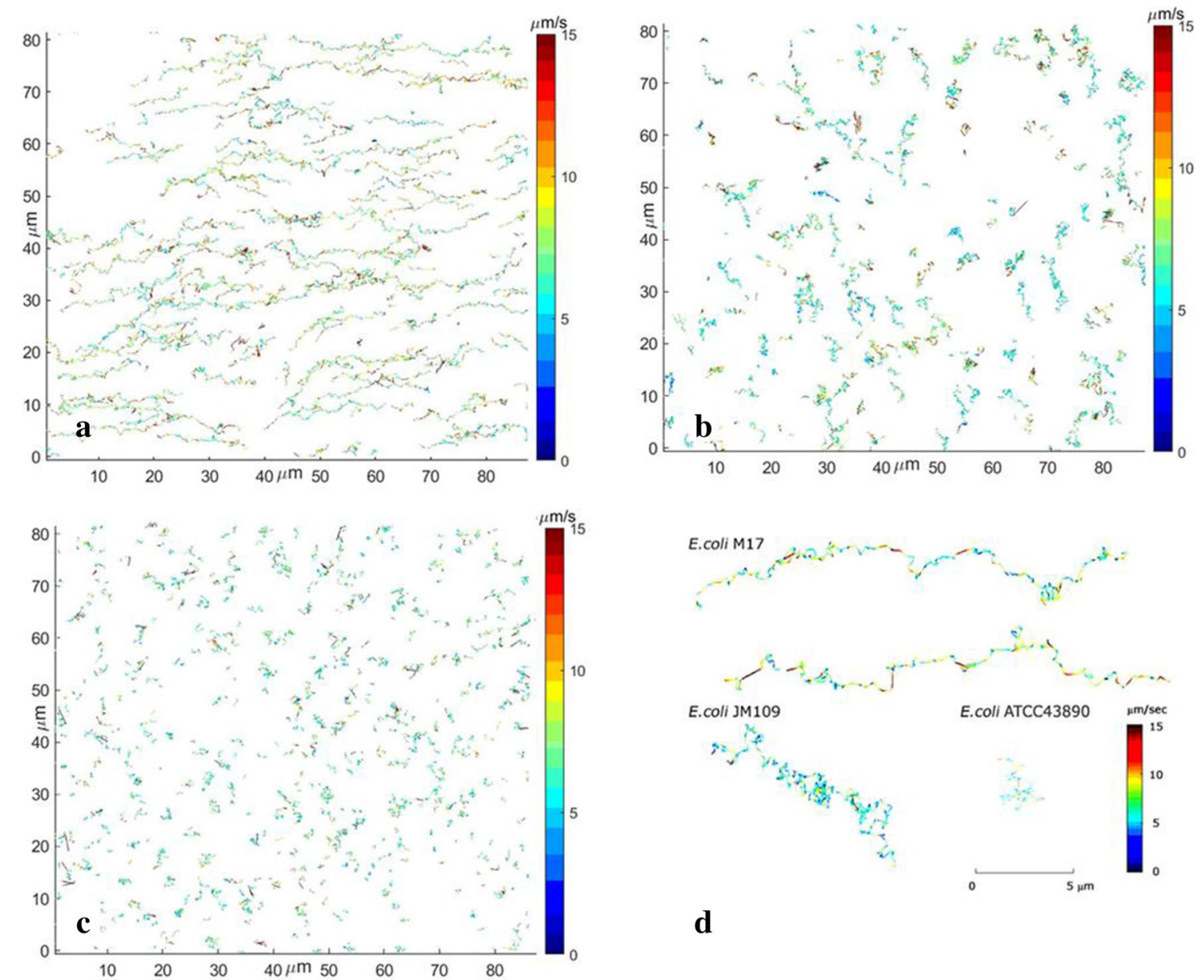

d
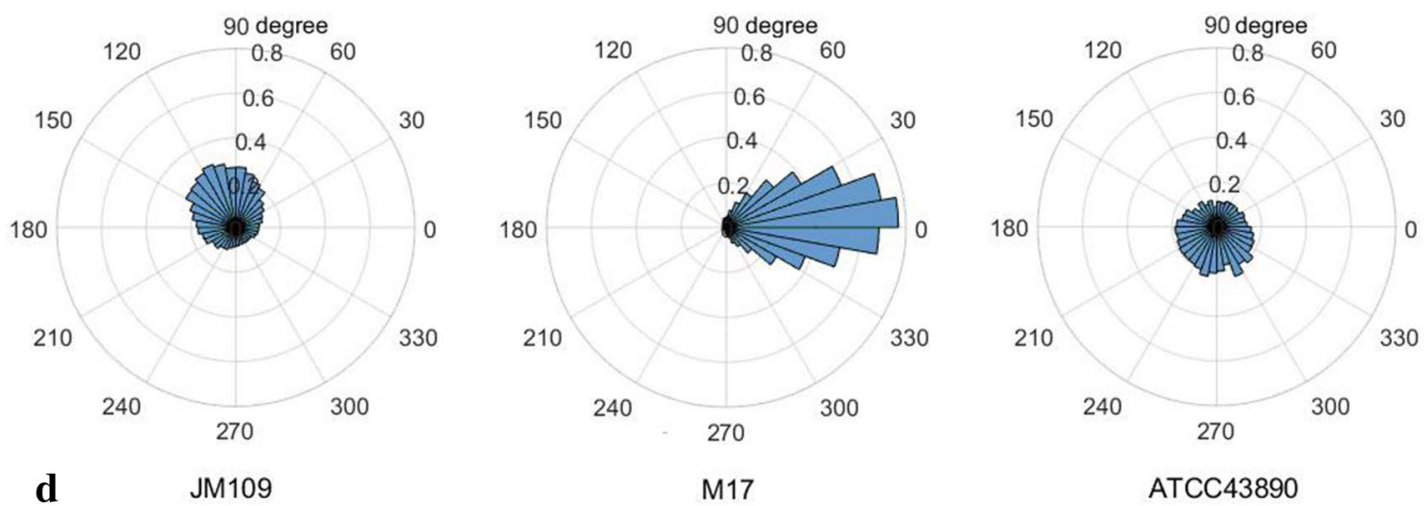

d

JM109

M17

ATCC43890

Figure 4. Reconstructed trajectories, color-coded according to the values of instantaneous velocities, for different strains of E. coli: (a) M17 (b) JM109 (c) ATCC43890. (d) Typical trajectory for each strain. (e) Angle distributions of instantaneous velocity vectors for each strain. M17 exhibits pronounced collective motion in one direction.

95\% 2.88-3.46), $7.01 \mu \mathrm{m}$ (CI 95\% 6.17-7.86), and $1.83 \mu \mathrm{m}$ (CI 95\% 1.65-2) for JM109, M17, and ATCC43890, respectively (Anova, $\mathrm{F}=81.54, p<0.01$ ). Interestingly, motile ATCC43890 showed almost a twice shorter mean distance than the poorly motile JM109. An average residence time was $3.62 \mathrm{~s}$ (CI 95\% 3.19-4.05), 2.99 s (CI 95\% 2.62-3.36), and $0.94 \mathrm{~s}$ (CI 95\% 0.84-1.04) for JM109, M17, and ATCC43890, respectively (Anova, F=51.5, $p<0.01$ ). The time spent within the observed layer by the M17 and JM109 strains was approximately the same, suggesting that both strains left the layer due to stochastic processes and gravity. Strain ATCC439890 demonstrated the shortest residence time compared to JM109 and M17 (Tukey, HSD = 2.68 (and 2.05), $p<0.01$ ). These data may be explained if we suggest that ATCC439890 preferred to 'scan' layer by layer, moving in a vertical direction more frequently than other strains. More statistical details one can see in a Supplementary. 


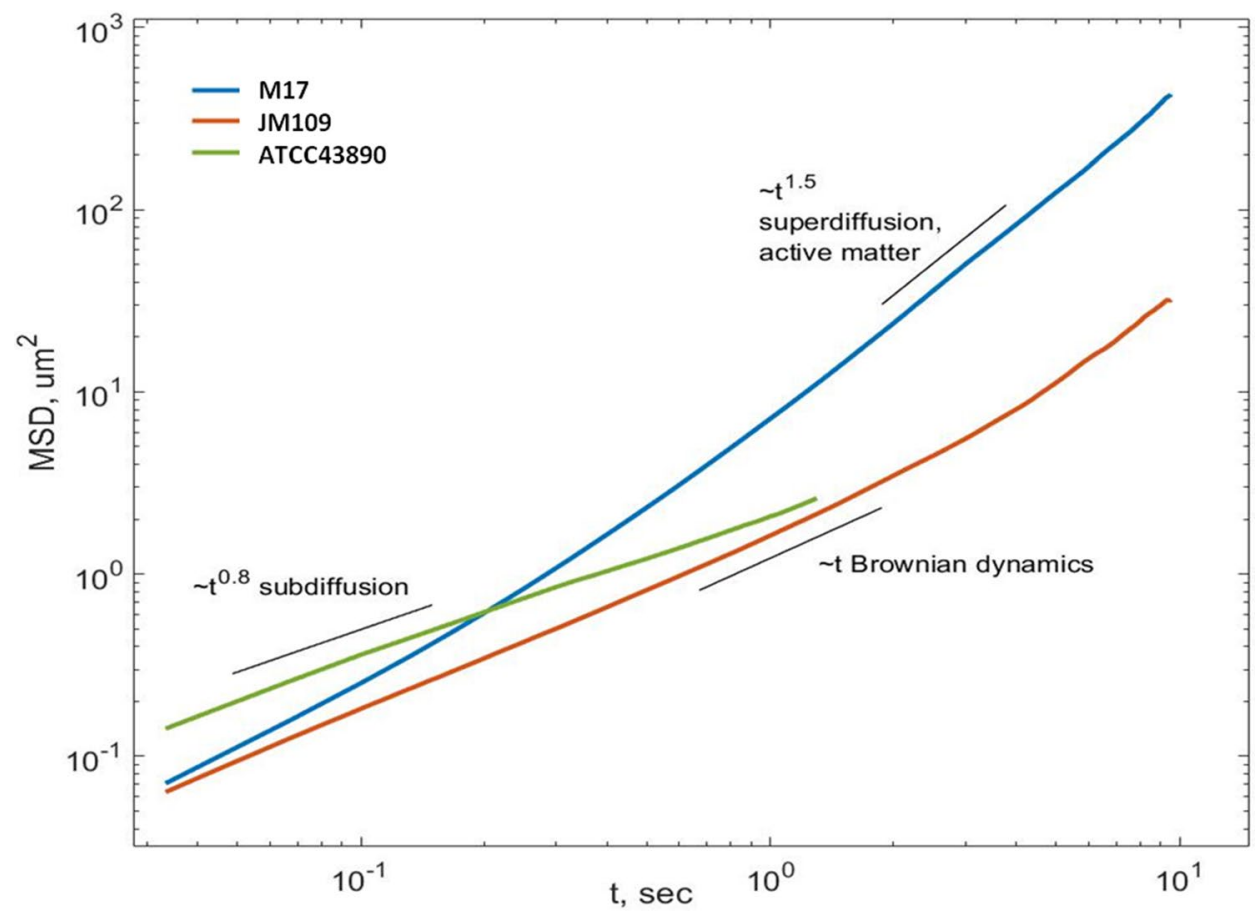

Figure 5. Log-log time dependence of mean square displacement (MSD) for various strains of E. coli.

Figure 4 shows $\sim 200$ bacteria trajectories obtained in one experiment that are color-coded according to the values of instantaneous velocities. The poorly motile JM109 strain demonstrated a typical Brownian-like motion, i.e., its chaotic movements were due to stochastic collisions with ambient molecules of liquid (Fig. $4 \mathrm{~b}, \mathrm{~d}$ ). The absence of a preferred movement direction is typical for Brownian particles as well ${ }^{23}$. M17 strain, on the contrary, had longer, less chaotic tracks with pronounced directional movement (Fig. 4a,d). At the same time, the ATCC43890 trajectories were the shortest, as if they had just crossed through the observed layer, and did not have a pronounced collective or individual directional movement, similar to JM109 (Fig. 4c,d). To check these observations, polar histograms, which show the direction of the instantaneous velocity vectors averaged over all bacteria, were constructed (Fig. 4e). The polar histograms showed that the population of M17 bacteria had a preferred instantaneous movement direction while other strains did not. These observations suggested that a noticeable part of the M17 bacteria moved in the same direction within each particular moment.

\section{E. coli suspension in terms of active matter: M17 demonstrated superdiffusive behavior while} ATCC43890 demonstrated subdiffusive behavior. To better understand the movement characteristics of each strain, we analysed bacterial behaviour in terms of the active matter theory ${ }^{24}$. From this point of view, bacterial suspension can be considered an active matter, that is, a system consisting of active particles capable of converting chemical energy to directional motion.

The analysis in terms of active matter theory is based on the calculation of the time $(t)$ dependence of the mean square displacement (MSD). This method reveals prevailing patterns of motion, varying from conventional Brownian (chaotic) motion up to the ballistic regime. Figure 5 shows a graph of $M S D(t)$ on a $\log -\log$ scale for the $E$. coli strains. Calculation of the MSD time dependence for the poorly motile strain JM109 revealed a linear MSD time dependence (Fig. 5). The linear $\operatorname{MSD}(t)$ dependence is characteristic of the motion of passive particles colliding with molecules of the medium, which is typical for classical Brownian dynamics ${ }^{23}$. For strain M17, the mean square displacement was proportional to the time taken to a power of $1.5\left(\sim \mathrm{t}^{1.5}\right)$. Such dependence indicates the superdiffusion of bacteria in the suspension ${ }^{24}$. Superdiffusion means that directional movement in the horizontal plane predominates over the chaotic component of motion, and M17 bacteria can be classified as active Brownian particles. These data are in line with the direct calculation of the average velocity direction vector (Fig. 4e).

The asymptotics for ATCC43890 turned out to be equal to MSD $\sim \mathrm{t}^{0.8}$ as if the bacteria had subdiffusive behaviour. However, we had to consider that the experimental design was restricted to observing bacteria in a horizontal layer $\sim 5 \mu \mathrm{m}$ thick. At the same time, the total height of the microfluidic camera was $30 \mu \mathrm{m}$, so the bacteria could quickly leave the field of visibility deep into the solution. In other words, our experimental design allowed observation of the horizontal motion only, while bacteria moved in the 3D medium, and subdiffusive bacterial motion in the horizontal direction might be compensated by superdiffusion in the vertical direction. The suggestion about the preferential movement of ATCC43890 bacteria in the vertical direction is supported by a short distance and short residence time in the horizontal layer (Fig. 4). 

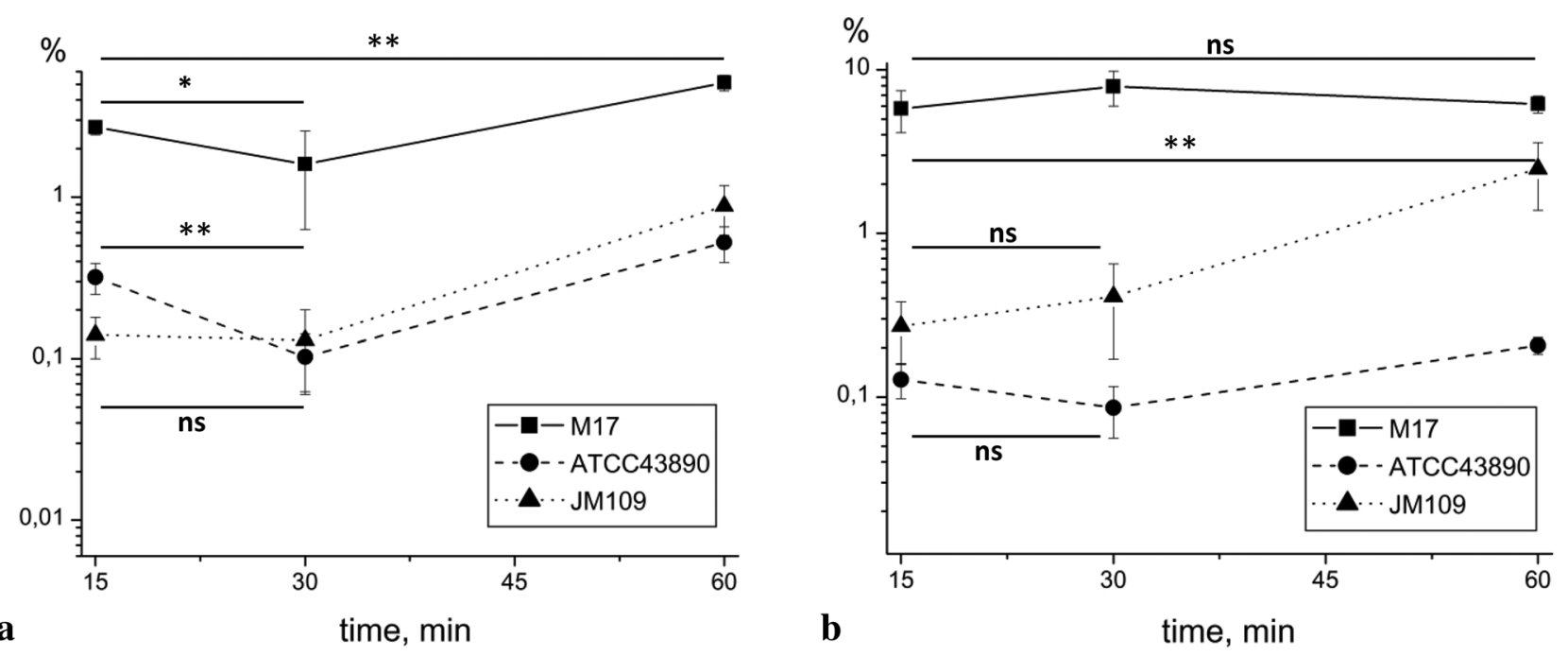

Figure 6. Efficiency of adhesion among different E. coli strains to (a) plastic and (b) HEp-2 cell line. ns-not significant, ${ }^{\star} p<0.05,{ }^{* *} p<0.01$. Additional information related to statistical analysis see in a Supplementary.

Therefore, the active matter analysis supported the individual characteristics of the motion of the studied $E$. coli strains. JM109 showed mostly Brownian movement, M17 moved collectively, representing active Brownian particle behaviour, while ATCC43890 moved highly likely in a vertical direction. Considering these noticeable differences in landing motility patterns between the studied strains, we were interested in how these microscopic characteristics would influence macroscopic parameters such as adhesion.

Motile and non-motile strains demonstrated different dynamics of adhesion to the plastic surface. Next, we compared the effectiveness of bacterial adhesion to horizontal surfaces in the three studied strains. Bacteria were added to a 24-well plate, and the number of adhered cells was determined after 15, 30, and $60 \mathrm{~min}$ of incubation. In $15 \mathrm{~min}$, poorly motile JM109 showed the lowest adhesion rate $(0.14 \pm 0.04 \%$ of initially added bacteria), but the prolongation of exposition to $60 \mathrm{~min}$ significantly increased the efficiency of its adhesion by a factor of $6.3 \pm 2.1$ times (Tukey, $\mathrm{HSD}=2.49, p<0.01$ ) (Fig. $6 \mathrm{a}$ ). Motile strains demonstrated more effective fast adhesion with adhered $2.71 \pm 0.2 \%$ and $0.32 \pm 0.07 \%$ for M17 and ATCC 43890 , respectively, 15 min post bacterial addition. However, the dynamics of motile strain adhesion were completely different from those of JM109. After 60 min of incubation, the absolute number of attached M17 bacteria doubled and reached $5.12 \pm 0.56 \%$ of the initial amount (Tukey, HSD $=2.31, p<0.01$ ). For ATCC43890, increasing the incubation time to 30 min resulted in a three-fold decrease in the number of attached cells from $0.32 \%$ to $0.1 \%$ (Tukey, $\mathrm{HSD}=0.22, p<0.01$ ), and $60 \mathrm{~min}$ incubation caused a $0.52 \%$ adhesion efficiency, which was the lowest among the three strains and 1.7 times lower than that of the poorly motile strain JM109. Thus, adhesion seemed to be a dynamic process for the motile strains and the result of a passive settlement for the poorly motile strain.

Differences in motility patterns resulted in different efficiency of adhesion to human HEp-2 cells. Both M17 and ATCC43890 strains can colonise the intestine as the first strain is probiotic, and the second is pathogenic. To analyse whether the observed differences in motility patterns influence bacterial interactions with epithelial cells, we tested bacterial adhesion to human adenocarcinoma HEp-2 cells, which are often used as models for $E$. coli infection ${ }^{25,26}$. They have laminin and fibronectin receptors, which are specific targets for the hemorrhagic E. coli strain ATCC $43890^{27}$. HEp-2 cells were grown up to a monolayer, and bacteria were added at a multiplicity of infection MOI of 1:100. Adhesion efficiency was tested 15, 30, and 60 min post infection by direct plating of adhered bacteria as described in the Materials and Methods.

After 15 min of incubation, adhesion rates were similar to the data obtained for the plastic surface for JM109 and M17, but not for ATCC43890 ( $0.27 \pm 0.11 \%$ and $5.79 \pm 1.56 \%$ for JM109 and M17, respectively) (Fig. 6b). ATCC43890 adhesion to HEp-2 cells was lower than to the plastic surface $(0.13 \pm 0.03 \%$ and $0.32 \pm 0.07 \%$ for HEp-2 and plastic surfaces 15 min post bacterial addition, respectively). Incubation for 60 min caused a 9.2 fold increase in the number of adhered JM109 bacteria compared to the first $15 \mathrm{~min}$ (Tukey, HSD $=2.31, p<0.01$ ). In contrast, prolonged incubation for 30-60 min did not increase the number of adhered M17 and ATCC43890 bacteria (1.07 and 1.5 fold increase, respectively, compared to bacteria adhered after 15 min incubation, $p>0.05$ ).

The results suggested that the increase in the adhesion efficiency of the poorly motile E. coli strain JM109 after 60 min of incubation was mainly due to bacterial sedimentation under the influence of gravity. The absence of positive dynamics for motile strains might suggest that the percentage of bacteria able to interact with human cells was limited, so such bacteria adhered to the cells during the first $15 \mathrm{~min}$, and later the population was exhausted. Alternatively, the number of available receptors on the cell surface may be restricted. Motile bacteria seized all receptors within $15 \mathrm{~min}$, and later, there were no free receptors for bacteria to adhere. The difference in adhesion dynamics to the plastic surface and HEp-2 cells supported the second suggestion: while the number of M17 and 

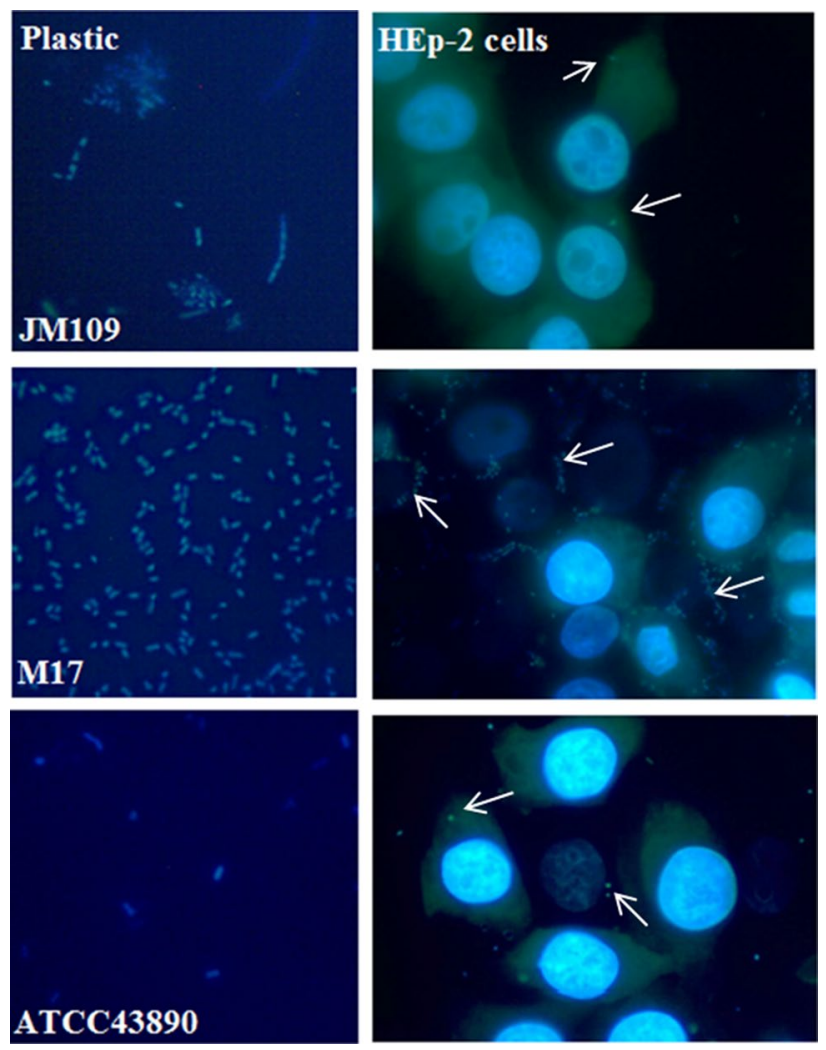

Figure 7. The microphotographs of adhered E. coli strains. Arrows show the adhered bacteria on specific locations of cell surfaces.

ATCC43890 bacteria adhered to the plastic surface increased from 15 to $60 \mathrm{~min}$, it was almost constant when bacteria interacted with HEp-2 cells.

Differences in motility patterns resulted in different patterns of adhesion. The obtained results demonstrated that the M17 and ATCC43890 strains demonstrated a noticeable difference in adhesion efficiency 15 min after bacterial addition. This difference was observed for adhesion to both plastic surfaces and human cells; therefore, this difference was not due to the varying abundance of strain-specific surface receptors used by bacteria to adhere to cells. We suggest that different motility patterns may affect the adhesion efficiency of motile strains.

To better understand the events during adhesion, we made microphotographs of bacteria adhered to plastic and HEp-2 cell surfaces after 15 min of incubation (Fig. 7). Poorly motile JM109 adhered to plastic and HEp-2 surfaces as small auto-aggregates that suggested initial flocculation, which is in line with our suggestion about passive sedimentation of JM109 cells (Fig. 7a,d). M17 demonstrated a non-uniform distribution on the plastic surface with linear clusters of 3-5 or more bacterial cells (Fig. 7b). Interacting with HEp-2 cells, M17 bacteria concentrated predominantly at the border of neighbouring cells in groups of more than ten bacteria with the same linear geometry as at the plastic surface (Fig. 7e). In contrast, ATCC43890 bacteria had completely different surface distributions. Single or groups of two bacteria were observed on the plastic surface, and mainly isolated bacteria were observed on the HEp-2 cell surface (Fig. 7c). ATCC43890 bacteria were distributed uniformly over surfaces, and there were no observed tropisms toward cell-cell contact areas for ATCC43890 bacteria, in contrast to M17 bacteria. The amount of E. coli ATCC43890 that adhered to the cells was higher than that of JM109, which contradicted the results of the plating experiment (see Fig. 6b). This discrepancy could be due to the weak adhesion strength of the ATCC43890 strain and the more delicate washing of the samples for taking microphotographs.

Taken together, the obtained results demonstrated noticeable differences between the motile strains not only in adhesion efficiency but also in surface distribution.

\section{Discussion}

In this study, three E. coli strains were compared from the point of view of specific features characterising bacteria leaving the bulk and approaching the solid surface. This initial stage of bacterial interactions with the surface, known as the landing stage, is important for further choice between adhesion to the surface or continuing the movement ${ }^{28}$. Three strains were compared: two motile strains, the probiotic strain M-17 and virulent O157:H7 
strain ATCC43890, and the poorly motile laboratory strain JM109. Bacterial motion patterns were studied at a distance of $10-15 \mu \mathrm{m}$ from the surface, which is a distance characteristic for the landing stage ${ }^{13}$.

The parameters of the average and maximal distances and residence time demonstrated by strain JM109 were considered characteristic of Brownian movement because JM109 is known to be poorly motile due to a deletion of the recA gene ${ }^{14-17}$. Low JM109 motility was confirmed by a macroscopic motility test. We expected that motile bacteria would move parallel to the surface, demonstrating longer average and maximal distances and a shorter residence time than JM109. This suggestion was based on the well-known models of bacterial landing and near-surface motility ${ }^{29,30}$. These models suggest that $E$. coli swimming at a distance of $20 \mu \mathrm{m}$ or less from the surface reorients the cells for their axis to be parallel to the surface due to hydrodynamic interactions ${ }^{29,30}$. Indeed, the probiotic strain M17 demonstrated this behaviour. M17 bacteria moved along lengthy, horizontally oriented trajectories.

Surprisingly, the probiotic strain M17 was the only strain that moved predictably in our experiments, while the motile virulent strain ATCC43890 did not demonstrate long trajectories. Moreover, ATCC43890 showed average and maximal distances and residence times shorter than the poorly motile strain JM109. These data suggest that ATCC43890 quickly left the horizontal liquid layer and moved preferentially in the vertical direction. Previously, Ipina et al. also used the enterohemorragic E.coli strain serotype O157:H7 to investigate its movement patterns near a glass surface ${ }^{12}$. They observed smooth circular trajectories due to the interruption of bacterial movement by short stop events ('transient adhesion events') ${ }^{12}$. It was concluded that these trajectories provided a faster exploration of a surface that could be profitable for all bacteria. We did not observe such behaviour because we focused on the landing stage, excluding the interaction of bacteria with a surface. Still, further observations on ATCC43890 movement with the techniques that allowed observation of the vertical motion are required to support the suggestion about preferable vertical motion direction.

The next parameter we analysed was the traits of the collective behaviour of bacteria. From a physical point of view, motile bacteria are active Brownian particles that can convert external energy into kinetic energy of motion $^{26-31}$. Based on this definition, the suspension of motile bacteria is a special case of active media that can be characterised by the formation of zones with aligned dynamic clusters, whirls, and jets, especially with increasing bacterial concentration ${ }^{32,33}$, similar to ${ }^{33,34}$ what we observed aligning the velocity vectors of M17 (Fig. 3). We did not find any published data on the subdiffusive behaviour of a motile bacterium, which was demonstrated by the strain ATCC43890; in addition, we failed to find data on a collective motion in a vertical direction that we suggested for this strain. This phenomenon requires further study.

We considered that the observed motility difference between probiotic and virulent strains could correlate with the efficiency of their adhesion to plastic and eukaryotic cells. Taken together, plating and microscopic data suggested that poorly motile JM109 tended to self-aggregate, form non-shaped flocs by a few cells, and floc sedimentation due to gravity. This result seems trivial. M17 adhesion in the form of linear formations was a more interesting observation. These linear formations were observed on both the plastic surfaces and the cell monolayer. When M17 bacteria interacted with a HEp-2 cell monolayer, the linear formation was strictly associated with the cell-to-cell border. Therefore, our first suggestion was that M17 bacteria specifically interacted with receptors located at the intercellular junctions. However, this suggestion was contradicted to the observation of similar structures formed on the plastic surfaces. To explain the linear patterns of M17 adhesion, we suggested that the linear character of adhesion was a direct consequence of the collective horizontal unidirectional movement demonstrated by this strain. We assumed that this collective unidirectional movement caused the formation of local 'braking zones' when interactions with a certain obstacle stopped the movement of at least one bacterium. Any obstacle that slows down or stops the movement of one bacterium should lead to the formation of a row of slow-moving or blocked bacteria, that is, an 'adhesion zone' (Fig. 7a,b). Such collective adhesion must increase the effectiveness of surface colonisation. Similar behaviour was observed for Salmonella Typhimurium ${ }^{7}$. Similar adhesion patterns were previously described for saprophytic E. coli strains by Frommel et al., who referred to it as a global adhesion strategy for commensal E. coli $i^{35}$.

The obstacles that stop bacterial rows can be quite minor irregularities on a plastic surface. When intercellular contact sites in the epithelial cell monolayer were considered, scanning electron microscopy revealed vertical edges formed by intercellular junctions ${ }^{36}$. Although these edges were approximately $200 \mathrm{~nm}$, this may be enough to slow down the speed of at least one bacterium because of the vertical character of the edge that prevents bacteria from moving around the obstacle. As a result, explosive growth in the number of bacteria in this zone can be observed. Therefore, we speculate that the horizontally oriented collective movement determines the characteristics of adhesion and the high adhesion efficiency.

The ATCC43890 bacteria demonstrated a different character of the movement and uniform distribution of single or double cells on plastic and cell surfaces. We suggest that this type of adhesion could be a consequence of the vertical character of the movement. Considering that virulent ATCC43890 requires a ligand-receptor interaction, this behaviour could be beneficial for the search of receptors. This 'vertical' movement may also be promising for intracellular pathogens. It has been shown, for example, that $S$. Typhimurium selects spherical mitotic cells for invasion ${ }^{7}$, and there is a correlation between the number of neighbouring eukaryotic cells and the efficiency of bacterial invasion ${ }^{37}$. The angle of interaction between bacteria and the surface is also important. For intestinal pathogens, the penetration of the mucus layer covering the surface of cells is critical, and motility facilitates this process ${ }^{38}$. It is evident that the shortest trajectory, in this case, is down through the protective layer at an angle close to $90^{\circ}$. Recently, Otte et al. showed that the active movement of $S$. Typhimurium, but not chemotaxis, was the primary strategy for the search of host cells because the adhesion was a random process depending on bacterial motility parameters ${ }^{39}$.

Thus, based on the data obtained, we propose two bacterial motility patterns for adequate adhesion: horizontal and vertical movements at the landing stage. The horizontal movement, characterised by a collective directed movement of microorganisms within the horizontal layer, could allow more effective non-specific 

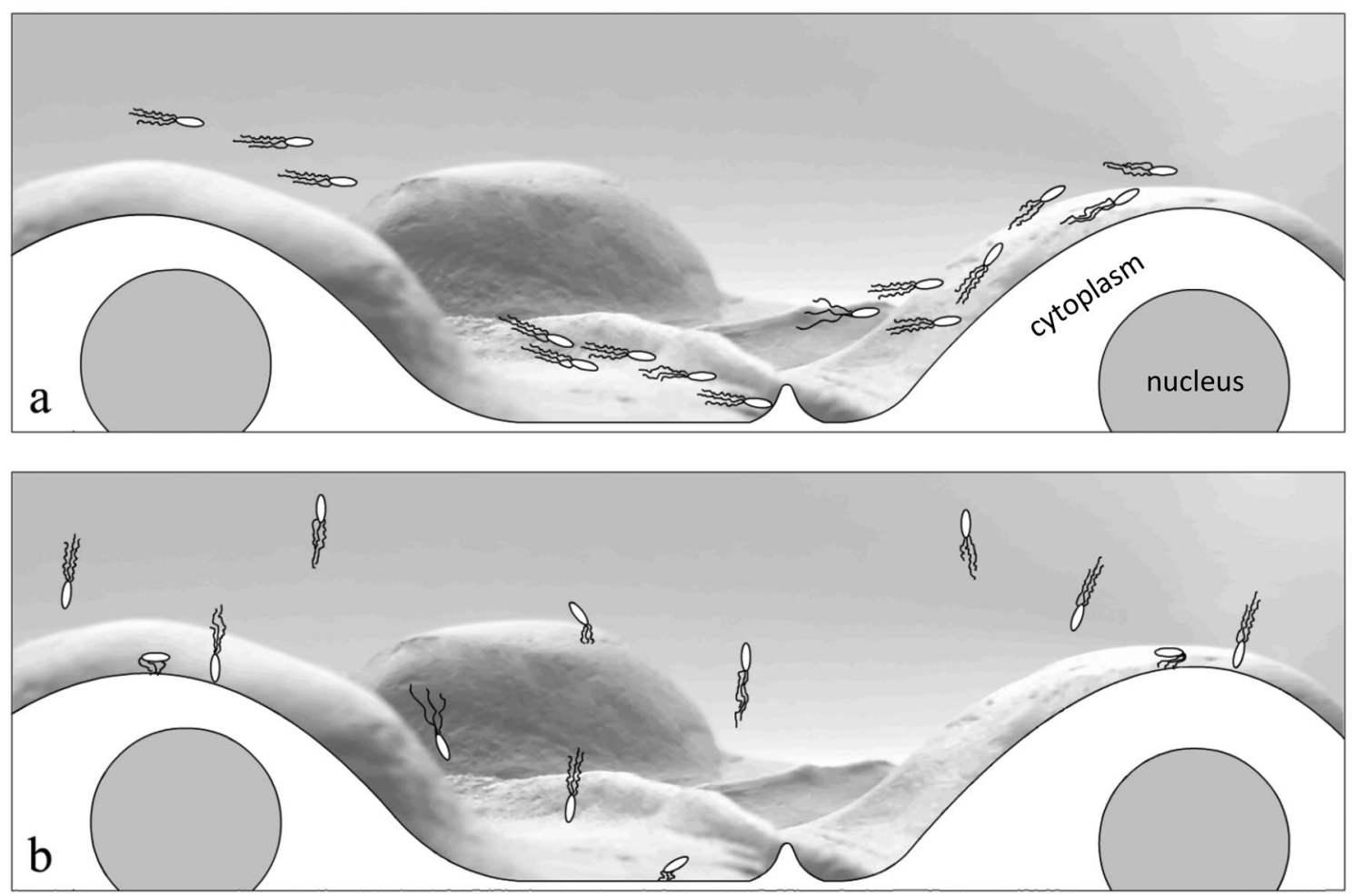

Figure 8. Horizontal (a) and vertical (b) movement for surface adhesion. Horizontal strategy implies collective directional motion mainly in the horizontal layer with gradual slow sedimentation and a non-uniform 'row' pattern of adhesion close to surface irregularities. Vertical strategy implies non-directional motion in the mainly vertical direction and uniformly distributed adhesion patterns.

surface colonisation. Movement in a vertical direction may promote a faster transition from bulk to near-surface swimming, allowing microorganisms to 'test' the surface and increase the probability of interaction with the receptor (Fig. 8). Therefore, individual differences in motility patterns may underlie different adhesion strategies.

\section{Materials and methods}

Bacterial strains and growth conditions. The investigation of bacterial movement was conducted with three E. coli strains from the Gamaleya National Research Centre collection: the enterohemorrhagic E. coli ATCC43890 strain ATCC43890, the probiotic strain E. coli M17, and E. coli JM109 with poor motility. Bacteria were routinely grown on Luria-Bertani (LB) agar (Amresco, USA) media at $37^{\circ} \mathrm{C}$.

Detection of bacterial motility on semi-solid agar. Semi-solid LB medium ( $0.5 \%$ agar) was dispensed in Petri dishes. Three E. coli strains were inoculated into semi-solid agar using a stab method with a straight needle. After $24 \mathrm{~h}$ incubation at $37^{\circ} \mathrm{C}$, a photograph of the Petri dish was taken, and the diameters of the opaque area were measured. This experiment was conducted in triplicates.

Microfluidic chamber construction. The prototype of the microfluidic chamber was a counting chamber that was modified by reducing the height of the medium layer to $30 \mu \mathrm{m}$ (Fig. 1). The chamber was created by 3D printing of a transparent resin (Anycubic Photon, China).

Observation of bacterial motility. For the investigation of bacterial movement, the liquid overnight culture that was grown in a shaking incubator at $37^{\circ} \mathrm{C}$ was centrifuged, and the sediment was resuspended in sterile phosphate-buffered saline (PBS) to reach an optical density of $2\left(\mathrm{OD}_{600}=2\right)$, which corresponded to a concentration of $1 \times 10^{9}$ cells $/ \mathrm{mL}$.

The bacterial suspension was inserted into the microfluidic chamber, followed by video visualisation at 1000-fold magnification. The chamber was placed onto the slide table of the Zeiss Axio Scope A1 microscope equipped with a digital camera DCM510 (China), which transmitted video images with a resolution of $1280 \times 960$ px (Fig. 2). The sample preparation and visual observation took approximately 10-15 $\mathrm{min}$, and then a series of $30 \mathrm{~s}$ videos were made at a speed of 30 frames/sec. The size of the observed field of view was $\sim 81 \times 86 \mathrm{~mm}^{2}$, and the depth of field was 5-10 microns.

At least five videos for each sample were recorded, and all experiments were conducted five times.

Video image processing and analysis. For the processing and analysis of videos, approximately $10 \mathrm{~s}$ of the video stream was cut out. We used the videos without obvious signs of movement of the bacteria due to a 
laminar flow to be sure that the bacteria move freely. he selected videos were processed using specially developed software Plasma 4.0, in which a statistical subtraction of the background, Fourier filtering of the image, and frame-by-frame identification of the location of each of the bacteria were performed. We considered trajectories longer than ten frames to cut off the 'false bacteria' blur circles with short trajectories of 1-10 frames in length that did not disappear during the preprocessing.

Thus, we obtained the main characteristics of the movement of bacteria: (1) positions of the bacteria in each frame, (2) instantaneous and average values of the speeds, (3) trajectory and mean square displacement, and (4) characteristic time and characteristic length of run of bacteria in the observing layer.

Adhesion of $\boldsymbol{E}$. coli strains to HEp-2 cells. HEp-2 cells were cultivated in Dulbecco's Modified Eagle Medium (DMEM; Paneco, Russia) supplemented with $10 \%$ fetal bovine serum (GIBCO) and no antibiotics. Cells were cultured at $37^{\circ} \mathrm{C}$ and $5 \% \mathrm{CO}_{2}$.

The cells were seeded in a 24-well plate. While achieving an average number, 300000 cells/well, the nutritious medium was replaced with $1 \mathrm{~mL}$ E. coli suspension at a ratio of 1:100. After 15, 30, and $60 \mathrm{~min}$ of incubation, the bacterial suspension was removed, and the surface of the wells was rinsed thrice with sterile PBS. To destroy the eukaryotic cells, PBS was removed, and $100 \mu \mathrm{l}$ of $1 \%$ Triton X-100 was added to the wells. After $15 \mathrm{~s}$, when the cell membranes disappeared, $900 \mu \mathrm{PBS}$ was added, and the suspension was resuspended thoroughly. After that, the samples were diluted and quantitated by plating on appropriate agar plates.

All the experiments were made in triplicate thrice.

Adhesion of $E$. coli strains to plastic. The adhesion efficiency was tested on a polyethylene terephthalate (PET) surface of 24-well plates. The same concentrations and volumes of bacterial suspension, as we used to investigate adhesion to HEp-2 cells, were added to each well of a 24-well plate. After 15, 30, and 60 min of incubation, the bacterial suspension was removed, and the surface of the wells was rinsed thoroughly thrice with sterile PBS. Bacteria were then removed from the surface with a sterile cotton swab. Tenfold dilutions were plated, and the number of colony-forming units (CFU) was counted after $18 \mathrm{~h}$ of incubation at $37^{\circ} \mathrm{C}$.

All the experiments were conducted in triplicate thrice.

Bacterial staining. Bacteria adhered to the plastic and HEp-2 cells were washed three times with sterile PBS, fixed with 3.7\% neutral formaldehyde, and stained with Hoechst 33,342 (ThermoFisher, USA) according to the manufacturer's protocol. Fluorescence microscopy and $1000 \times$ magnification were used to visualise the samples.

Statistical analysis. Values are expressed as mean \pm SD. Statistical analysis was performed using a oneway ANOVA with post hoc Tukey's test. Statistical differences were considered significant when the $p$-value was $<0.05$.

Received: 19 July 2021; Accepted: 22 December 2021

Published online: 12 January 2022

\section{References}

1. Siryaporn, A., Kuchma, S. L., O’Toole, G. A., Gitai, Z. \& Ausubel, F. M. Surface attachment induces Pseudomonas aeruginosa virulence. Proc. Natl. Acad. Sci. U. S. A. 111, 16860-16865 (2014).

2. Wolfson, E. B. et al. The interaction of Escherichia coli O157:H7 and Salmonella Typhimurium flagella with host cell membranes and cytoskeletal components. Microbiology (United Kingdom) 166, 947-965 (2020).

3. Ottemann, K. M. \& Miller, J. F. Roles for motility in bacterial-host interactions. Mol. Microbiol. 24, 1109-1117 (1997).

4. Josenhans, C. \& Suerbaum, S. The role of motility as a virulence factor in bacteria. Int. J. Med. Microbiol. 291, 605-614 (2002).

5. O’Neil, H. S. \& Marquis, H. Listeria monocytogenes flagella are used for motility, not as adhesins, to increase host cell invasion. Infect. Immun. 74, 6675-6681 (2006).

6. Belas, R. Biofilms, flagella, and mechanosensing of surfaces by bacteria. Trends Microbiol. 22, 517-527 (2014).

7. Misselwitz, B. et al. Near surface swimming of salmonella Typhimurium explains target-site selection and cooperative invasion. PLoS Pathog. 8, 9 (2012).

8. Molaei, M., Barry, M., Stocker, R. \& Sheng, J. Failed escape: Solid surfaces prevent tumbling of Escherichia coli. Phys. Rev. Lett. 113, 1-6 (2014).

9. Makarchuk, S., Braz, V. C., Araújo, N. A. M., Ciric, L. \& Volpe, G. Enhanced propagation of motile bacteria on surfaces due to forward scattering. Nat. Commun. 10, 4110 (2019).

10. Spagnolie, S. E. \& Lauga, E. Hydrodynamics of self-propulsion near a boundary: Predictions and accuracy of far-field approximations. J. Fluid Mech. 700, 105-147 (2012).

11. Lauga, E., DiLuzio, W. R., Whitesides, G. M. \& Stone, H. A. Swimming in circles: Motion of bacteria near solid boundaries. Biophys. J. 90, 400-412 (2006).

12. Perez Ipiña, E., Otte, S., Pontier-Bres, R., Czerucka, D. \& Peruani, F. Bacteria display optimal transport near surfaces. Nat. Phys. 15, 610-615 (2019).

13. Qi, M., Gong, X., Wu, B. \& Zhang, G. Landing dynamics of swimming bacteria on a polymeric surface: effect of surface properties. Langmuir 33, 3525-3533 (2017).

14. Pratt, L. A. \& Kolter, R. Genetic analysis of Escherichia coli biofilm formation: Roles of flagella, motility, chemotaxis and type I pili. Mol. Microbiol. 30, 285-293 (1998).

15. Lymberopoulos, M. H. et al. Characterization of Stg fimbriae from an avian pathogenic Escherichia coli O78:K80 strain and assessment of their contribution to colonization of the chicken respiratory tract. J. Bacteriol. 188, 6449-6459 (2006).

16. Mayola, A. et al. RecA protein plays a role in the chemotactic response and chemoreceptor clustering of Salmonella enterica. PLoS ONE 9, e1055578 (2014). 
17. Frutos-Grilo, E., Marsal, M., Irazoki, O., Barbé, J. \& Campoy, S. The interaction of RecA with both CheA and CheW is required for Chemotaxis. Front. Microbiol. 11, 1-15 (2020).

18. Zhou, M. et al. Long polar fimbriae contribute to pathogenic Escherichia coli infection to host cells. Appl. Microbiol. Biotechnol. 103, 7317-7324 (2019).

19. Vergara, A., Vidal, R., Torres, A. G. \& Farfan, M. J. Long polar fimbriae participates in the induction of neutrophils transepithelial migration across intestinal cells infected with enterohemorrhagic E. coli O157:H7. Front. Cell. Infect. Microbiol. 4, 1-6 (2014).

20. Farfan, M. J. \& Torres, A. G. Molecular mechanisms that mediate colonization of shiga toxin-producing Escherichia coli strains. Infect. Immun. 80, 903-913 (2012).

21. Torres, A. G., Zhou, X. \& Kaper, J. B. Adherence of diarrheagenic. Microbiology 73, 18-29 (2005).

22. Beutin, L. et al. Genetical and functional investigation of fliC genes encoding flagellar serotype $\mathrm{H} 4$ in wildtype strains of Escherichia coli and in a laboratory E. coli K-12 strain expressing flagellar antigen type H48. BMC Microbiol. 5, 1-11 (2005).

23. Dini, C., Bolla, P. A. \& de Urraza, P. J. Treatment of in vitro enterohemorrhagic Escherichia coli infection using phage and probiotics. J. Appl. Microbiol. 121, 78-88 (2016).

24. Bechinger, C. et al. Active particles in complex and crowded environments. Rev. Mod. Phys. 88, 045006 (2016).

25. McWilliams, B. D. \& Torres, A. G. Enterohemorrhagic Escherichia coli adhesins. Microbiol. Spectr. 2, (2014).

26. Mathewson, J. J. \& Cravioto, A. HEp-2 cell adherence as an assay for virulence among diarrheagenic Escherichia coli. J. Infect. Dis. 159, 1057-1060 (1989).

27. Einstein, A. Über die von der molekularkinetischen Theorie der Wärme geforderte Bewegung von in ruhenden Flüssigkeiten suspendierten Teilchen. Ann. Phys. 4, (1905).

28. Berne, C., Ellison, C. K., Ducret, A. \& Brun, Y. V. Bacterial adhesion at the single-cell level. Nat. Rev. Microbiol. 16, 616-627 (2018).

29. Berke, A. P., Turner, L., Berg, H. C. \& Lauga, E. Hydrodynamic attraction of swimming microorganisms by surfaces. Phys. Rev. Lett. 101, 1-4 (2008).

30. Desai, N. \& Ardekani, A. M. Biofilms at interfaces: Microbial distribution in floating films. Soft Matter 16, 1731-1750 (2020).

31. Selmeczi, D. et al. Cell motility as random motion: A review. Eur. Phys. J. Spec. Top. 157, 1-15 (2008).

32. Sidortsov, M., Morgenstern, Y. \& Be’Er, A. Role of tumbling in bacterial swarming. Phys. Rev. E 96, 1-7 (2017).

33. Sokolov, A., Aranson, I. S., Kessler, J. O. \& Goldstein, R. E. Concentration dependence of the collective dynamics of swimming bacteria. Phys. Rev. Lett. 98, 1-4 (2007).

34. Wioland, H., Lushi, E. \& Goldstein, R. E. Directed collective motion of bacteria under channel confinement. New J. Phys. 18, 075002 (2016)

35. Frömmel, U. et al. Adhesion patterns of commensal and pathogenic Escherichia coli from humans and wild animals on human and porcine epithelial cell lines. Gut Pathog. 5, 1-8 (2013).

36. Brückner, B. R. \& Janshoff, A. Importance of integrity of cell-cell junctions for the mechanics of confluent MDCK II cells. Sci. Rep. 8, 1-11 (2018).

37. Voznica, J., Enninga, J. \& Stévenin, V. High-throughput microscopic analysis of salmonella invasion of host cells. Bio-Protocol. 8, (2018).

38. Furter, M., Sellin, M. E., Hansson, G. C. \& Hardt, W. D. Mucus architecture and near-surface swimming affect distinct salmonella typhimurium infection patterns along the murine intestinal tract. Cell Rep. 27, 2665-2678.e3 (2019).

39. Otte, S., Ipiña, E. P., Pontier-Bres, R., Czerucka, D. \& Peruani, F. Statistics of pathogenic bacteria in the search of host cells. Nat. Commun. 12, 1-9 (2021).

\section{Acknowledgements}

We thank Dr. Valentina Pushkareva of the laboratory of ecology of pathogenic bacteria of N. F. Gamaleya National Research Centre of Epidemiology and Microbiology, Ministry of Health of the Russian Federation (Moscow) for the kindly provided E. coli ATCC43890 strain. This study was funded by grant RFBR №20-34-90127.

\section{Author contributions}

E.S.A., S.Y.M., S.E.V., P.O.F. designed the experiments. A.M.M., V.E.V., S.E.V., G.A.I., D.P.A., S.D.A., V.M.M. performed the experiments and analyzed the data. E.S.A., S.E.V., A.M.M., V.E.V., S.Y.M. wrote the manuscript. All authors have approved the final version of the manuscript.

\section{Competing interests}

The authors declare no competing interests.

\section{Additional information}

Supplementary Information The online version contains supplementary material available at https:/doi.org/ 10.1038/s41598-021-04592-y.

Correspondence and requests for materials should be addressed to E.V.S.

Reprints and permissions information is available at www.nature.com/reprints.

Publisher's note Springer Nature remains neutral with regard to jurisdictional claims in published maps and institutional affiliations.

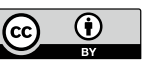

Open Access This article is licensed under a Creative Commons Attribution 4.0 International License, which permits use, sharing, adaptation, distribution and reproduction in any medium or format, as long as you give appropriate credit to the original author(s) and the source, provide a link to the Creative Commons licence, and indicate if changes were made. The images or other third party material in this article are included in the article's Creative Commons licence, unless indicated otherwise in a credit line to the material. If material is not included in the article's Creative Commons licence and your intended use is not permitted by statutory regulation or exceeds the permitted use, you will need to obtain permission directly from the copyright holder. To view a copy of this licence, visit http://creativecommons.org/licenses/by/4.0/.

(C) The Author(s) 2022 